CARDIOVASCULAR MEDICINE

\title{
Pulsed wave Doppler tissue echocardiography assessment of the long axis function of the right and left ventricles during the early neonatal period
}

\author{
K Mori, R Nakagawa, M Nii, T Edagawa, Y Takehara, M Inoue, Y Kuroda
}

Heart 2004;90:175-180. doi: 10.1136/hrt.2002.008110

See end of article for authors' affiliations

.....................

Correspondence to:

Dr K Mori, Department of

Paediatrics, School of

Medicine, University of

Tokushima, 3-18-15

Kuramoto-cho, Tokushima

City, Tokushima, 770-

8503, Japan; tonmaru@

clin.med.tokushima-u.ac.jp

Accepted 9 June 2003

\begin{abstract}
Objective: To assess the long axis function of both ventricles during the early neonatal period by using pulsed wave Doppler tissue (PWDT) echocardiography.

Design: PWDT echocardiography was recorded from the lateral sites of the mitral and tricuspid annuluses and the tip of interventricular septum in 130 neonates within 24 hours after birth (day 0 group), in 135 neonates 1-7 days after birth (day 1-7 group), and in 131 healthy children (children group).

Results: Peak systolic motion velocity (Sw) of the three ventricular walls positively correlated with the number of days after birth $(p<0.005)$. Compared with the children group, in neonates $S w$ in the right ventricle and peak early diastolic motion velocity $(\mathrm{Ew})$ and peak atrial systolic motion velocity in the interventricular septum were lower than in the remaining two walls $(p<0.0005, p<0.0001$, and $p<0.0001$, respectively). Although peak mitral and tricuspid flow velocities during early diastole (E) correlated with the number of hours after birth in the day 0 group, there was no significant change in the Ew of either ventricle. The E:Ew ratio of both ventricles was significantly higher in both neonate groups than in the children group $(p<0.001)$. The E:Ew ratio of the left ventricle was higher in the day 0 group than in the day $1-7$ group $(p<0.005)$.

Conclusions: The two ventricles differ in their normal PWDT echocardiographic values and in the parameter change after birth during the early neonatal period, which may reflect differences in ventricular adaptation after birth.
\end{abstract}

S ignificant haemodynamic changes occur during transition from the fetal to the neonatal environment. ${ }^{1}$ The ductus arteriosus closes and pulmonary blood flow increases in association with decreased pulmonary vascular resistance. Consequently, the preload of the left ventricle increases, and the increased systemic arterial blood pressure caused by the closed placental circulation increases the afterload of the left ventricle. In the right ventricle, the afterload gradually decreases because pulmonary hypertension is attenuated. In addition to the changes in loading conditions on the heart, the contractility and histological structure of the ventricular myocardium change after birth. Neonatal myocardium develops less force than the adult myocardium, and myofibrillar and sarcoplasmic reticulum contents increase after birth. ${ }^{23}$

Pulsed Doppler echocardiography of both tricuspid and mitral flows is used to evaluate the ventricular diastolic filling non-invasively. There are several reports concerning changes in the mitral and tricuspid flow patterns during the neonatal period. ${ }^{4-6}$ On the other hand, tissue Doppler echocardiography allows the recording of myocardial contraction and relaxation velocity. Pulsed wave Doppler tissue (PWDT) echocardiography can be used to analyse the long axis function of both ventricles, which is difficult to evaluate by conventional echocardiographic methods. ${ }^{7-12}$

We recently reported normal values of left ventricular wall motion velocity in healthy children, as measured with PWDT echocardiography. ${ }^{13}$ There are no reports, however, of the normal value for PWDT echocardiographic measurements of right and left ventricles during the early neonatal period. It is not clear whether PWDT parameters change in accordance with significant haemodynamic change and histological maturation of the myocardium during the early neonatal period. The purposes of this study were to establish normal values for both ventricular wall motion velocities along the longitudinal direction with the use of PWDT echocardiography during the first seven days after birth, and to clarify the relation between mitral and tricuspid flow patterns and diastolic PWDT parameters.

\section{METHODS}

\section{Study population}

Informed parental consent was obtained for each of the 230 term newborns (mean (SD) gestational age 39.6 (1.4) weeks) who took part in the study. Mean (SD) birth weight was 3002 (329) g. In some neonates, echocardiographic examination was performed serially, for a total of 265 echocardiograms. The ages of the neonates ranged from 0-7 days. Because loading conditions of both ventricles change significantly during the first 24 hours after birth, ${ }^{5-6}$ the neonate group was divided into two subgroups: neonates within 24 hours after birth ( $\mathrm{n}=130$, day 0 group) and neonates from 24 hours to seven days after birth ( $\mathrm{n}=135$, day 1-7 group). Physical examination was normal and two dimensional echocardiography showed no cardiac defects. Persistent ductus arteriosus was considered normal up to day 3. In all babies, heart rate was between 100-145 beats/min. Table 1 shows clinical and conventional echocardiographic data of the two subgroups of neonates. For comparison, 131 healthy infants and children ranging from 2 months to 19 years of age were also examined (mean (SD) age 7.5 (5.5) years) (children group).

Abbreviations: $A$, peak atrial flow velocity; $A w$, peak atrial systolic motion velocity; $\mathrm{E}$, peak early diastolic flow velocity; $\mathrm{Ew}$, peak early diastolic motion velocity; PWDT, pulsed wave Doppler tissue; Sw, peak systolic motion velocity 
Table 1 Clinical and conventional echocardiographic data in two neonate groups

\begin{tabular}{|c|c|c|c|}
\hline & $\begin{array}{l}\text { Day } 0 \text { group } \\
(n=130)\end{array}$ & $\begin{array}{l}\text { Day 1-7 group } \\
(n=135)\end{array}$ & $p$ Value \\
\hline Age (after birth) & $10.2(7.2)$ hours & $3.9(2.0)$ days & \\
\hline Gestational age (weeks) & $39.7(1.1)$ & $39.5(1.6)$ & NS \\
\hline Heart rate (beats $/ \mathrm{min}$ ) & $125(10)$ & $125(8)$ & NS \\
\hline Weight (g) & $3055(321)$ & $2947(330)$ & $<0.01$ \\
\hline LV diastolic dimension (mm) & $17.5(1.7)$ & $17.2(1.6)$ & NS \\
\hline Shortening fraction of LV & $0.31(0.06)$ & $0.31(0.05)$ & NS \\
\hline Transtricuspid E $(\mathrm{cm} / \mathrm{s})$ & $38.2(6.9)$ & $37.9(7.1)$ & NS \\
\hline Transtricuspid A $(\mathrm{cm} / \mathrm{s})$ & $49.2(8.4)$ & $48.9(7.5)$ & NS \\
\hline Transtricuspid A:E & $1.31(0.23)$ & $1.32(0.25)$ & NS \\
\hline Transmitral E (cm/s) & $56.2(10.3)$ & $52.1(9.5)$ & $<0.001$ \\
\hline Transmitral A $(\mathrm{cm} / \mathrm{s})$ & $50.0(7.6)$ & $48.2(8.0)$ & NS \\
\hline Transmitral A:E & $0.91(0.17)$ & $0.95(0.20)$ & NS \\
\hline
\end{tabular}

Values are mean (SD).

$A$, peak atrial flow velocity; $E$, peak early diastolic flow velocity; LV, left ventricle; NS, not significant.

Some of the PWDT echocardiography data in the children group has already been reported. ${ }^{13}$

\section{Echocardiographic study}

Echocardiographic examination was performed with an Aloka SSD-5500 (Aloka Corp, Tokyo, Japan) with 5 or 3.75 MHz transducers. All neonates were examined while they were lying quietly in the right anterior oblique position breathing room air. To record mitral and tricuspid flow velocities with the pulsed Doppler method, the sample volume was placed at the tip of both valve leaflets in the apical four chamber view. The peak atrial flow velocity (A), the peak early diastolic flow velocity (E), and their ratio (A:E) were determined.

Myocardial wall motion velocity was recorded by activating the PWDT function on the same machine with a sample volume of $2 \mathrm{~mm}$ and a pulse repetition frequency of $1.5 \mathrm{kHz}$. In the apical four chamber view, the sample volumes were placed in the lateral sites of the mitral and tricuspid annuluses and the base of the interventricular septum (fig 1). Because the angle between the estimated direction of wall motion and the Doppler beam was less than $20^{\circ}$, the Doppler signal angle was not corrected.

On the basis of the wall motion velocity patterns that were obtained, the peak systolic ( $\mathrm{Sw}$ ), peak early diastolic (Ew), and peak atrial systolic (Aw) motion velocities were measured. Although Sw was often separated into two components ( $\mathrm{S} 1 \mathrm{~W}$ and $\mathrm{S} 2 \mathrm{~W}$ ) in the interventricular septum and left lateral wall in most of the children group, ${ }^{11}$ that of the neonate groups had one peak in all cases.

Pulsed Doppler echocardiographic data were obtained on line and recorded simultaneously with the ECG and respiration curve. Strip chart recordings of Doppler velocities were obtained at $50 \mathrm{~mm} / \mathrm{s}$ with a time resolution of $5 \mathrm{~ms}$. The mean values for three heart beats during expiration were used for the analysis.

\section{Statistical analysis}

Values are expressed as mean (SD). The mean values for each PWDT variable obtained from the various walls were compared by analysis of variance and Dunn's test. To determine the relation between PWDT variables or mitral or tricuspid flow velocities and days after birth, a simple linear regression analysis was used.

To test the intra-observer and inter-observer reproducibilities, Bland-Altman analysis was used. ${ }^{14}$ After a five minute rest period, repeated measurements were taken by observer 1 , observer 2, and again by observer 1. In total, 60 ventricular walls from 20 neonates were analysed. Variability was also expressed as the mean percentage error, derived as the absolute difference between the two sets of observations, divided by the mean of the observations. A probability value of $\mathrm{p}<0.05$ was considered significant.

\section{RESULTS}

\section{Normal PWDT values in neonates}

Transmitral E was significantly higher in the day 0 group than in the day $1-7$ group $(\mathrm{p}<0.001)$, although remaining Doppler blood flow parameters were not significantly different between the two neonate groups (table 1).
A

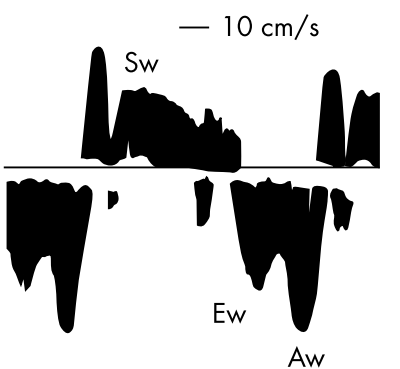

Aw
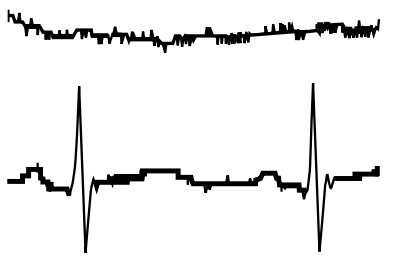

B $\quad-10 \mathrm{~cm} / \mathrm{s}$
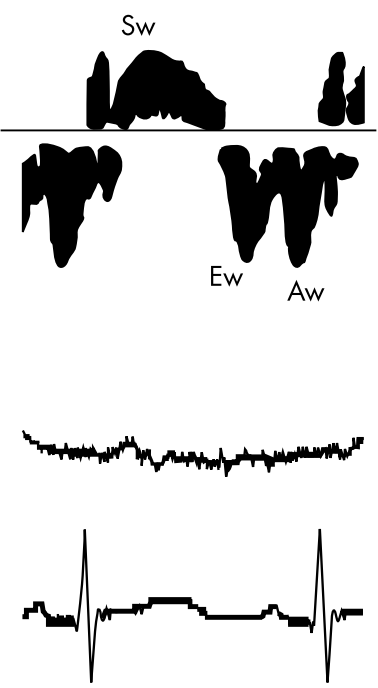

C $\quad-10 \mathrm{~cm} / \mathrm{s}$
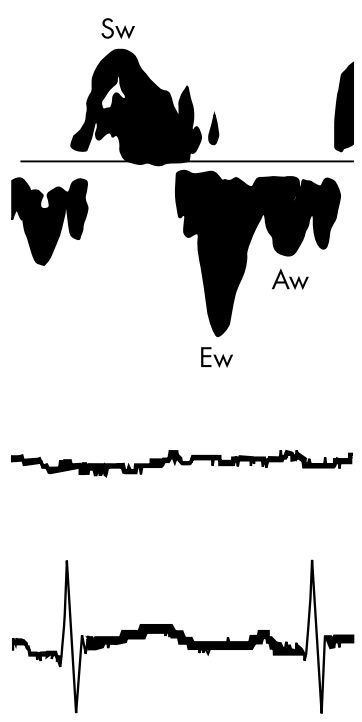

Figure 1 Representative recordings of the velocity spectrum of the wall motion assessed by pulsed wave Doppler tissue echocardiography in a baby two days after birth in the apical four chamber view. Recordings from (A) the tricuspid annulus, (B) the base of the ventricular septum, and (C) the mitral annulus. The corresponding ECG and respiration curve were simultaneously recorded. Aw, peak atrial systolic motion velocity; Ew, peak early diastolic motion velocity, LA, left atrium; LV, left ventricle; RA, right atrium; RV, right ventricle; Sw, peak systolic motion velocity. 
Table 2 Pulsed wave Doppler tissue velocities at each position in each phase of the cardiac cycle

\begin{tabular}{|c|c|c|c|}
\hline & RV & IVS & LV \\
\hline \multicolumn{4}{|c|}{ Day 0 group $(n=130)$} \\
\hline $\mathrm{S}_{w}(\mathrm{~cm} / \mathrm{s})$ & $6.2(1.1)$ & $3.7(0.6)^{*} \dagger$ & $5.3(0.9) \ddagger$ \\
\hline $\mathrm{Ew}(\mathrm{cm} / \mathrm{s})$ & $7.5(1.4)$ & $5.0(1.0)^{*} \dagger$ & $7.5(1.5)$ \\
\hline$A w(\mathrm{~cm} / \mathrm{s})$ & $9.2(1.6)$ & $4.9(1.0) * \dagger$ & $6.2(1.4) \ddagger$ \\
\hline Aw:Ew & $1.26(0.29)$ & $1.01(0.27)^{*} \dagger$ & $0.86(0.26) \ddagger$ \\
\hline \multicolumn{4}{|c|}{ Day 1-7 group $(n=135)$} \\
\hline $\mathrm{Sw}_{\mathrm{w}}(\mathrm{cm} / \mathrm{s})$ & $6.6(1.2)$ & $3.9(0.7)^{*} \dagger$ & $5.5(0.8) \ddagger$ \\
\hline $\mathrm{Ew}(\mathrm{cm} / \mathrm{s})$ & $7.5(1.3)$ & $5.4(0.9)^{*} \dagger$ & $7.7(1.5)$ \\
\hline$A w(\mathrm{~cm} / \mathrm{s})$ & $9.2(1.5)$ & $5.2(0.9)^{*} \dagger$ & $6.5(1.2) \ddagger$ \\
\hline Aw:Ew & $1.25(0.27)$ & $1.00(0.22)^{*} \dagger$ & $0.88(0.23) \ddagger$ \\
\hline \multicolumn{4}{|c|}{ Children group $(n=131)$} \\
\hline $\operatorname{SIw}(\mathrm{cm} / \mathrm{s})$ & $13.7(2.0)$ & $6.7(1.4)^{*} \dagger$ & $9.3(2.8) \ddagger$ \\
\hline $\mathrm{S} 2 \mathrm{w}(\mathrm{cm} / \mathrm{s})$ & & 7.5 (1.3)§ & $7.0(1.2)$ \\
\hline $\mathrm{Ew}(\mathrm{cm} / \mathrm{s})$ & $15.8(2.9)$ & $13.5(2.4)^{*} \dagger$ & $18.1(4.1) \ddagger$ \\
\hline$A w(\mathrm{~cm} / \mathrm{s})$ & $9.9(2.7)$ & $6.5(1.4)^{*} \S$ & $6.2(1.6) \ddagger$ \\
\hline$A w: E w$ & $0.65(0.21)$ & $0.50(0.18)^{*} \dagger$ & $0.36(0.14) \ddagger$ \\
\hline \multicolumn{4}{|c|}{$\begin{array}{l}\text { Data are mean (SD). } \\
\text { * } \mathrm{p}<0.0001, \mathrm{RV} v \mathrm{IVS} ; \mathrm{tp}<0.0001, \mathrm{IVS} v \mathrm{LV} ; \mathrm{fp}<0.0001, \mathrm{RV} v \mathrm{LV} \text {; } \\
\S \mathrm{s}<0.05 \text {, IVS } v \mathrm{LV} \text {. } \\
\text { Aw, peak atrial systolic motion velocity; Ew, peak early diastolic motion } \\
\text { velocity; IVS, interventricular septum recorded from its base; LV, left } \\
\text { ventricle recorded from lateral site of the mitral annulus; RV, right } \\
\text { ventricle recorded from lateral site of the tricuspid annulus; Sw, peak } \\
\text { systolic motion velocity. In the children group, two peak waveforms(SIw, } \\
\mathrm{S} 2 \mathrm{w} \text { ) were noted during systole in the IVS and LV wall. }\end{array}$} \\
\hline
\end{tabular}

Table 2 shows mean peak PWDT velocities for all three ventricular walls. In both neonate groups, interventricular septum PWDT velocities were significantly lower than those

Day 0 group $(n=130)$
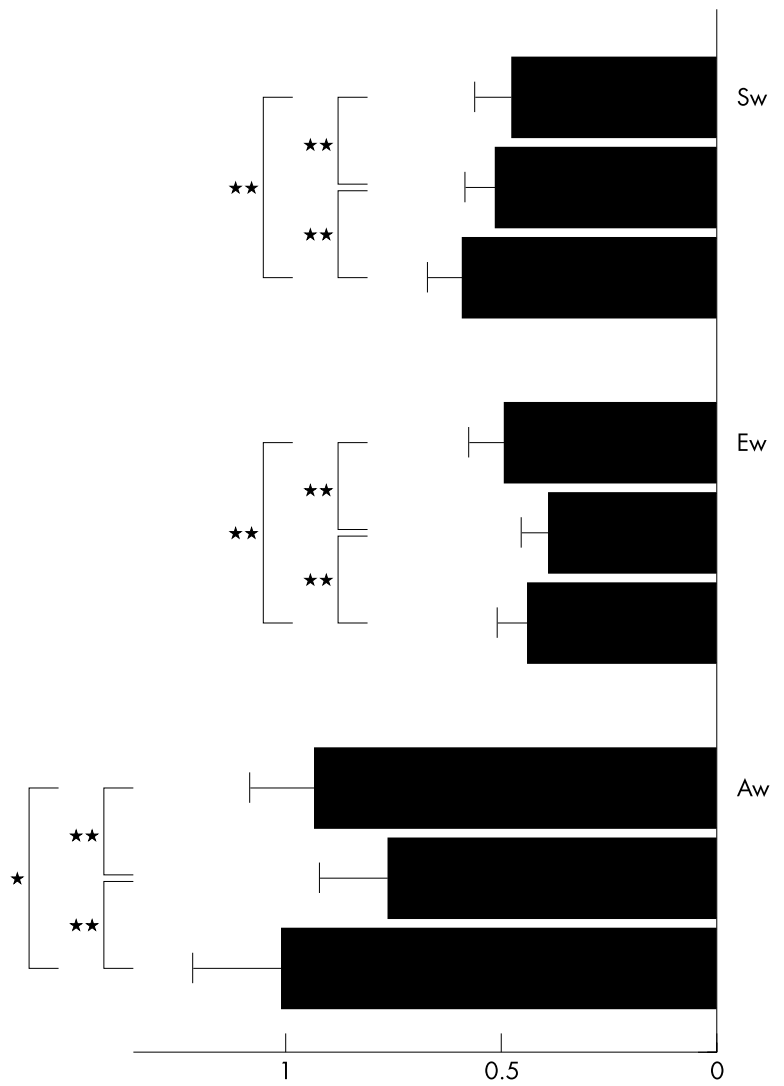

Ew of the remaining ventricular walls in any phase of the cardiac cycle $(\mathrm{p}<0.0001)$. In the right ventricular wall, Aw was the largest component of three PWDT variables in both neonate groups $(\mathrm{p}<0.0001)$. While Aw and Ew were similar and were higher than $\mathrm{Sw}$ in the interventricular septum $(p<0.0001)$, Ew was the largest component in the left ventricle $(\mathrm{p}<0.0001)$.

To compare the PWDT velocity of the neonate groups with that of the children group, each neonate's PWDT velocity was expressed as a ratio to the mean value of the peak PWDT velocity of the children group (fig 2). During systole, Sw of the right ventricle was lower than that of the remaining two walls in both neonate groups $(p<0.0005)$. During diastole, Ew and Aw of the interventricular septum were lower than those of the remaining two walls in both neonate groups $(p<0.0001)$. Ew of the neonate groups was less than half that of the children group in all of the ventricular walls. In contrast, Aw was more than three quarters that of the children group in all three ventricular walls. These results indicate that the wall motion velocity during the early neonatal period is relatively low in the right ventricle during systole and in the interventricular septum during diastole, suggesting that adaptation of these regions may be delayed after birth.

\section{Changes in PWDT parameters during the early neonatal period}

Sw of all three ventricular walls in the day 1-7 group positively correlated with the numbers of days after birth $(r=0.30$ in the right ventricle, $r=0.25$ in the

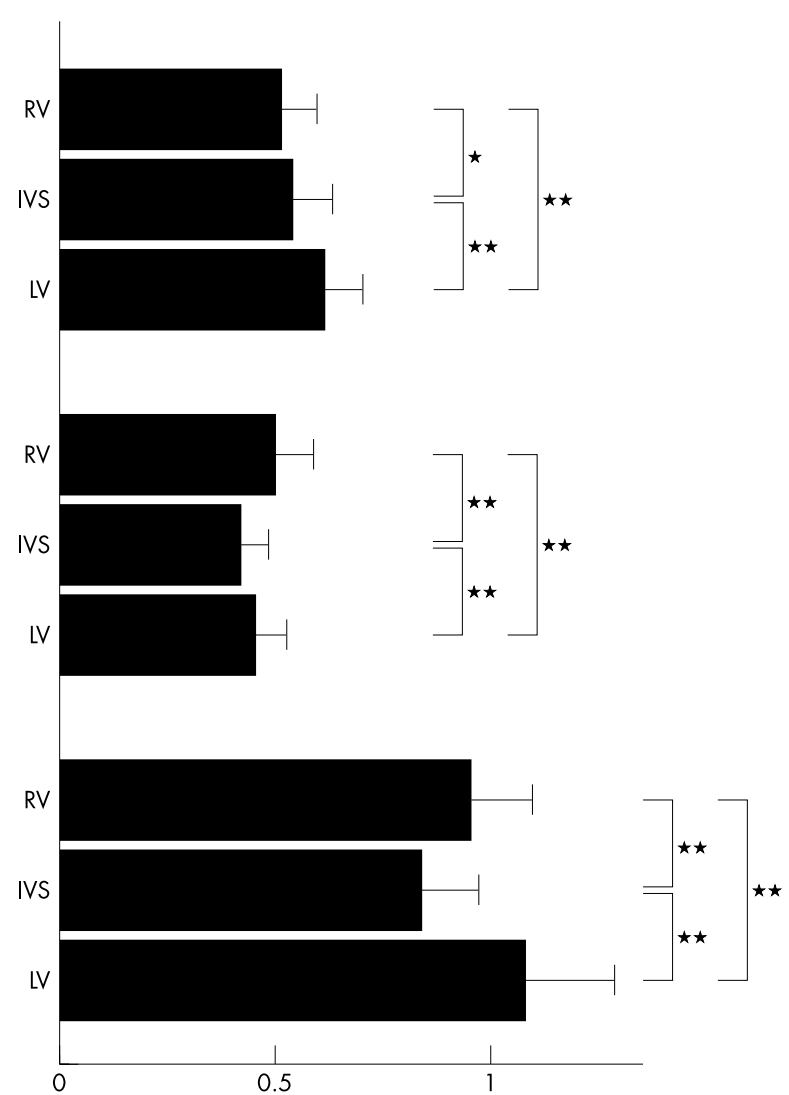

Figure 2 Ratio of pulsed wave Doppler tissue velocities at three ventricular walls of neonate groups to those of the children group. The PWDT velocity at three ventricular walls in each phase of the cardiac cycle of each neonate was expressed as a ratio to the mean value of peak PWDT velocity of the children group. RV was recorded from lateral site of the tricuspid annulus, IVS (interventricular septum) from its base, and LV from the lateral site of the mitral annulus. ${ }^{*} \mathrm{p}<0.0005,{ }^{* *} \mathrm{p}<0.0001$. 

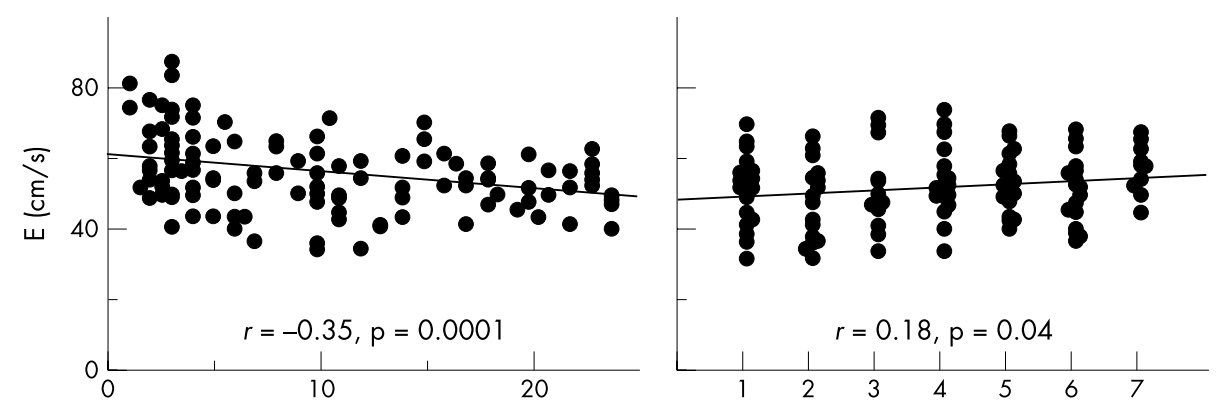

Figure 3 Relation between left ventricular Doppler parameters during early diastole and age after birth. Left panels are day 0 group and right panels day 1-7 group. $X$ axis (age) is number of hours after birth in the day 0 group and days after birth in the day 1 to 7 group. $Y$ axis is peak mitral early diastolic flow velocity (E) (upper panels), Ew in the mitral annulus (middle panels), and the E:Ew ratio
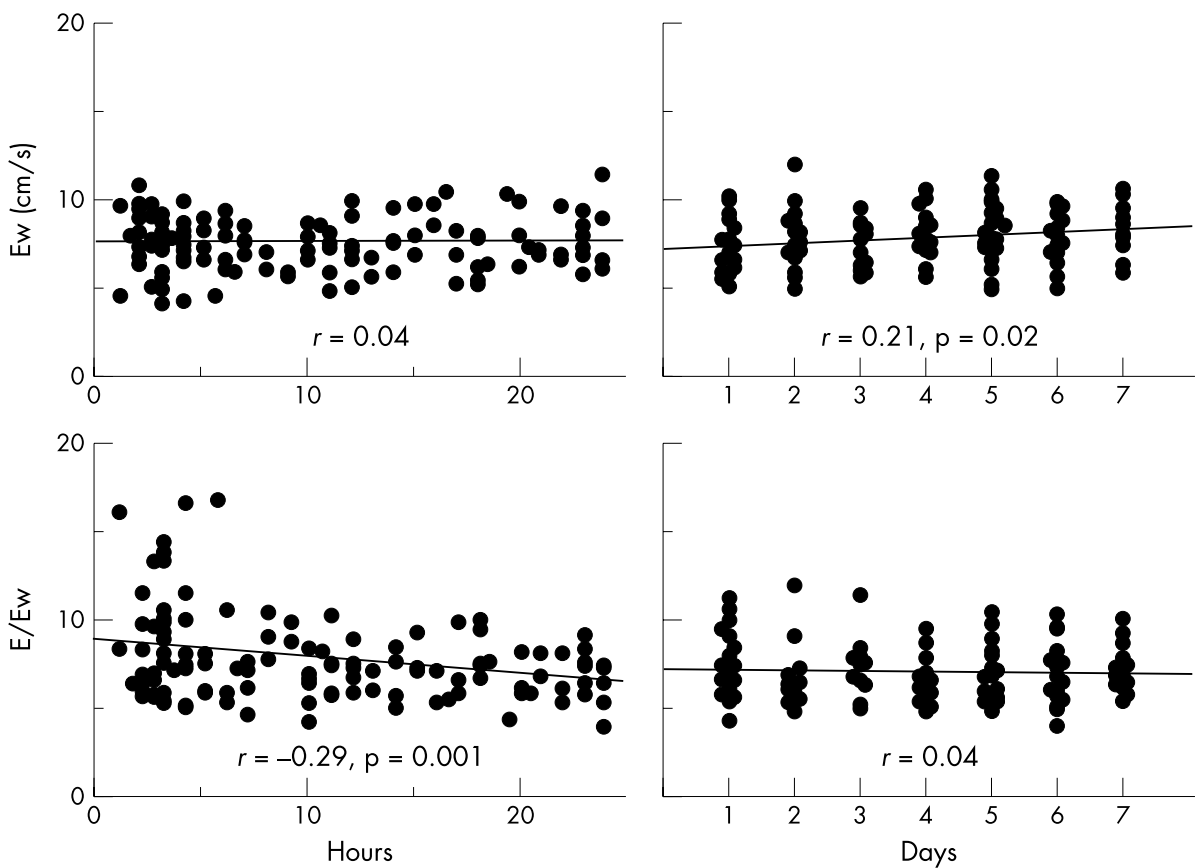

interventricular septum, and $r=0.29$ in the left ventricle; all $\mathrm{p}<0.005)$.

During the first 24 hours after birth, Ew of the three ventricular walls did not correlate significantly with the number of hours after birth, although peak mitral and tricuspid E did $(\mathrm{p}<0.0001$ and $\mathrm{p}<0.005$, respectively $)$ (figs 3 and 4 ). In the day 1-7 group, mitral $E$ increased according to the number of days after birth $(p<0.05)$. Ew of the interventricular septum and the left ventricle also correlated positively with the number of days after birth (both $\mathrm{p}<0.05$ ). Neither peak tricuspid E nor Ew of the right ventricular wall correlated significantly with number of days after birth.

Neither Aw of three ventricular walls nor tricuspid and mitral A correlated significantly with number of hours (or days) after birth. The mitral A:E ratio was lower than the tricuspid A:E ratio in both neonate groups (both $\mathrm{p}<0.0001$ ). Similarly, the Aw:Ew ratio was largest in the right ventricle and smallest in the left ventricle among the three ventricular walls in both neonate groups (both $\mathrm{p}<0.0001$ ) (table 2).

\section{E:Ew ratio during the neonatal period}

In the left ventricle, the transmitral E:Ew ratio decreased significantly during the first 24 hours after birth $(p=0.001)$ and thereafter the E:Ew ratio did not change significantly during the first week after birth (fig 3). The mean E:Ew in the left ventricle in the day 0 group was significantly larger than that in the day $1-7$ group $(7.8$ (2.4) $v 7.0$ (1.6), $\mathrm{p}=0.001)$. In contrast, the E:Ew ratio of the right ventricle in both the day 0 and day $1-7$ groups had no significant relation with the number of hours or days after birth, respectively (fig 4$)$. The mean values of the two groups did not differ significantly (5.2 (1.1) v $5.2(1.2))$.

The E:Ew ratio in the children group was $5.6(1.7)$ in the left ventricle and $3.4(0.8)$ in the right ventricle. These values were lower for both ventricles than in the two neonate groups (both $\mathrm{p}<0.001$ ). In any age groups, the E:Ew ratio of the left ventricle was higher than that of the right ventricle (all $\mathrm{p}<0.001)$.

\section{Reproducibility of PWDT echocardiography}

In the Bland-Altman test, the standard deviation of the interobserver and intra-observer difference of each PWDT parameter ranged from $0.32-0.61 \mathrm{~cm} / \mathrm{s}$.

The mean inter-observer error of Sw, Ew, and Aw was 7.9 $(4.5) \%, 7.6(4.9) \%$, and $7.9(5.2) \%$, respectively. The mean intra-observer error of Sw, Ew, and Aw was $5.2(3.0) \%, 5.0$ (3.6)\%, and $5.5(3.6) \%$, respectively.

\section{DISCUSSION}

\section{Systolic wall motion velocity}

With regard to longitudinal axis function of the adult heart, Sw of the tricuspid annulus is higher than that recorded from the mitral annulus, probably because the right ventricle has a lower afterload than the left and the role of right ventricular inflow is more predominant in right ventricular ejection than in the infundibular part. ${ }^{15}$ In fact, Sw of the right lateral wall was 1.5 times higher than that of the left lateral wall in the 

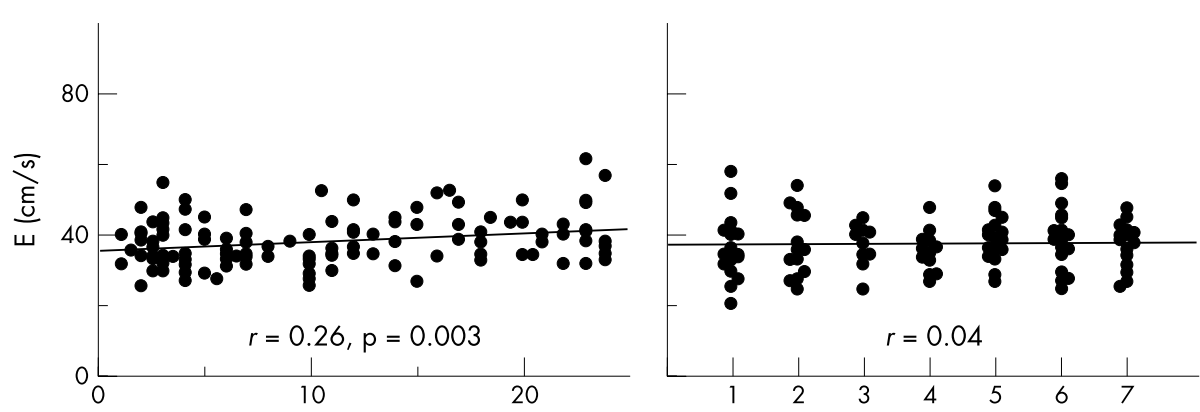

Figure 4 Relation between RV Doppler parameters during early diastole and age after birth. Left panels are day 0 group and right panels day 1-7 group. $X$ axis (age) is number of hours after birth in the day 0 group and days after birth in the day 1-7 group. $Y$ axis was tricuspid $E$ (upper panels), Ew in the tricuspid annulus (middle panels), and the E:Ew ratio (lower panels).
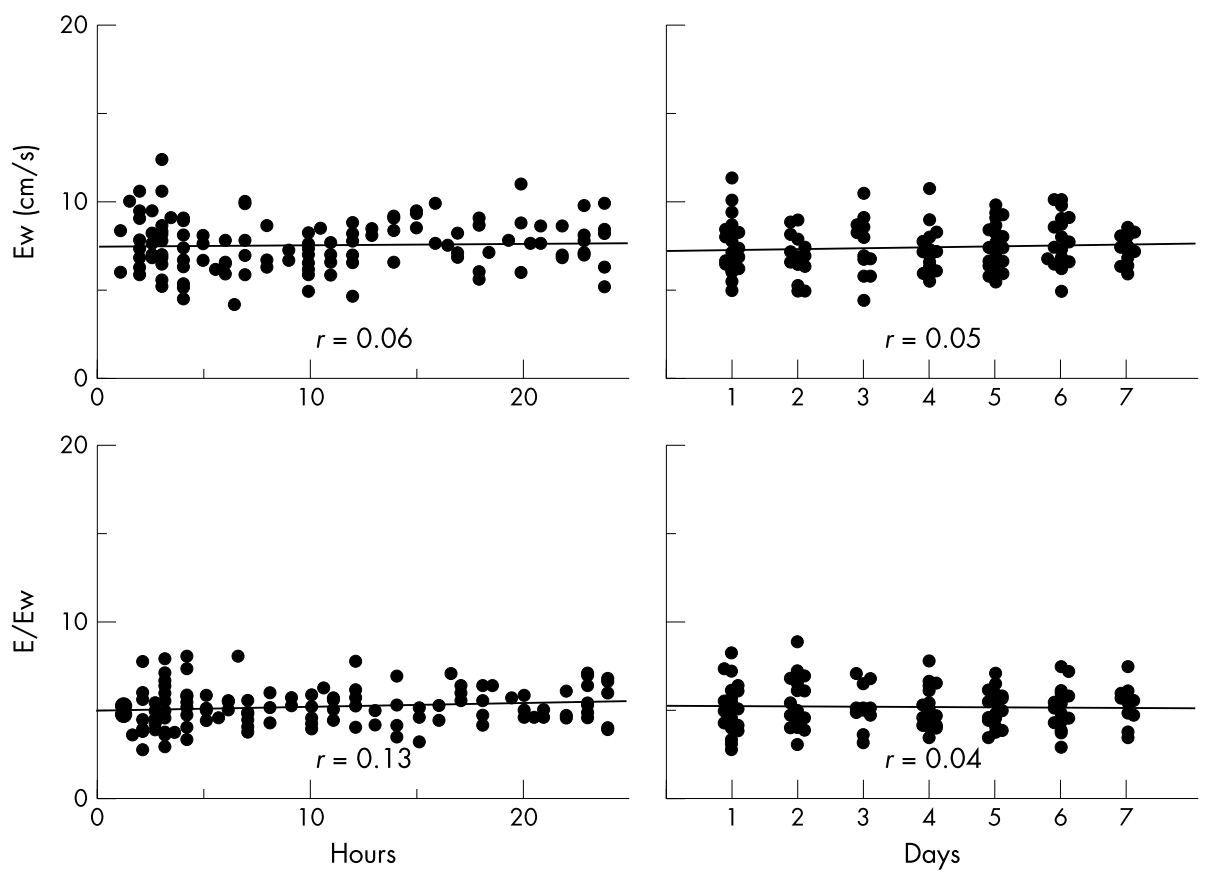

children group. On the other hand, Sw of the right lateral wall in both neonate groups was only 1.2 times higher than that in the left lateral wall. This attenuation of right ventricular predominance of longitudinal myocardial movement during systole is probably a result of the increased afterload caused by physiological pulmonary hypertension and immature contractile properties of the right ventricle itself. Sw was not separated into two components ( $\mathrm{S} 1 \mathrm{w}$ and S2W) in the neonate groups, probably because of the increased heart rate and limited time resolution of Doppler recordings by the echo equipment used in this study.

\section{Diastolic wall motion velocity}

During the first 24 hours after birth, the peak mitral E decreases because of a reduction in the preload resulting from closure of the ductus arteriosus. ${ }^{5}$ On the other hand, Ew of the mitral annulus did not change significantly. Harada and colleagues $^{6}$ reported that tricuspid E increases within 24 hours after birth, probably due to an increase in the right ventricular preload and decreased right ventricular afterload. Ew of the tricuspid annulus did not change significantly during this period, unlike Ew of the mitral annulus. To be precise, Ew is influenced by preload conditions. ${ }^{16}$ However, our results indicate that Ew of the three ventricular walls was influenced less by changes in the loading conditions observed during the first 24 hours after birth than by tricuspid or mitral flow.

After the decrease in mitral E during the first 24 hours after birth, there is a gradual increase as the number of days after birth increases. ${ }^{17}$ Concomitantly, Ew of both the mitral annulus and the interventricular septum increased, probably due to the development of diastolic function of the left ventricle. In contrast, neither tricuspid E nor Ew of the tricuspid annulus changed significantly during this period. These results suggest that development of diastolic function of the right ventricle may be delayed after birth relative to that of the left ventricle.

Frommelt and colleagues ${ }^{18}$ showed that the Aw in both ventricular lateral walls in infants less than 1 year old was similar to that of older children, although both Sw and Ew were significantly lower. Our data were the same as in their study: Aw during the early neonatal period was similar to that of the children group. The Aw:Ew ratio was higher in the tricuspid annulus than in the mitral annulus, indicating that relaxation may be impaired in the right ventricle.

\section{E:Ew ratio}

There are only a few reports of the E:Ew ratio in paediatric populations. Harada and colleagues ${ }^{19}$ reported that the E:Ew ratio of the left ventricle could be used to estimate the left atrial pressure in patients with an ventricular septal defect or persistent ductus arteriosus. In normal adults, the E:Ew ratio of the left ventricle is reported to be 7.7 (3.0). ${ }^{9}$ In our study, the children group had a lower E:Ew ratio than do adults, probably indicating lower left ventricular filling pressure. On the other hand, the E:Ew ratio was higher in the day 0 group, indicating a relatively higher left ventricular filling pressure caused by persistent ductus arteriosus or immature diastolic 
property of the myocardium. With the closure of the ductus arteriosus, the E: Ew ratio of the left ventricle decreased in the day 1-7 group.

The E:Ew ratio of the right ventricle is positively correlated with mean right atrial pressure. ${ }^{11}$ In contrast to the E:EW ratio of the left ventricle, that of the right ventricle did not change significantly during the early neonatal period and thereafter it decreased to the level of the children group. Our data indicate that the right ventricular filling pressure is also relatively high for neonatal myocardium and adaptation may be delayed compared with the left ventricle.

\section{Study limitations}

There are several limitations to this study. Firstly, the spatial orientation of myocardial fibres and their motion patterns follow complex three dimensional models. Some degree of angulation between the Doppler beam and the true direction of myocardial movement may exist, especially in the analysis of both mitral and tricuspid annuluses. Although this angulation may be small, it should be pointed out that the data presented in this study are for velocities along the Doppler beam direction and may not indicate a true myocardial velocity. Secondly, because PWDT echocardiography has the limitation that a stationary sample volume is positioned on a moving target, the effect of translation is not eliminated. Thirdly, because PWDT parameters are influenced by loading conditions, ${ }^{16}$ the data obtained from this study do not reflect intrinsic contractility or diastolic property but rather the regional cardiac movement for a given loading condition.

\section{Conclusion}

The current study establishes normal values of PWDT parameters during the early neonatal period. Further studies are required to validate the clinical usefulness of PWDT measurements in neonates with various cardiac dysfunctions.

\section{Authors' affiliations}

K Mori, R Nakagawa, M Nii, T Edagawa, Y Takehara, M Inove,

Y Kuroda, Department of Paediatrics, School of Medicine, University of

Tokushima, Tokushima City, Japan

\section{REFERENCES}

1 Rudolph AM. Congenital diseases of the heart. Chicago: Year Book Medicine Publishers, 1974:17-26.

2 Nakanishi T, Okuda H, Kamata K, et al. Development of myocardial contractile system in the fetal rabbit. Pediatr Res 1987;22:201-7.

3 Stopfkuchen $\mathrm{H}$. Changes of the cardiovascular system during the perinatal period. Eur J Pediatr 1987;146:545-9.

4 Riggs TW, Rodriguez R, Snider AR, et al. Doppler echocardiographic evaluation of right and left ventricular diastolic function in normal neonates. J Am Coll Cardiol 1989;13:700-5.

5 Harada K, Shiota T, Takahashi Y, et al. Doppler echocardiographic evaluation of left ventricular output and left ventricular diastolic filling changes in the first day of life. Pediatr Res 1994;35:506-9.

6 Harada K, Shiota T, Takahashi Y, et al. Right ventricular diastolic filling in the first day of life. Tohoku J Exp Med 1994;172:227-35.

7 Gulati VK, Katz WE, Follandsbee WP, et al. Mitral annular descent velocity by tissue Doppler echocardiography as an index of global left ventricular function. Am J Cardiol 1996;77:979-84

8 Ueti OM, Camargo EE, Ueti Ade A, et al. Assessment of right ventricular function with Doppler echocardiographic indices derived from tricuspid annular motion: comparison with radionuclide angiography. Heart 2002;88:244-8.

9 Shon DW, Chai IH, Lee DJ, et al. Assessment of mitral annulus velocity by Doppler tissue imaging in the evaluation of left ventricular diastolic function. J Am Coll Cardiol 1997:30:474-80.

10 Nagueh SF, Middleton KJ, Kopelen HA, et al. Doppler tissue imaging: a noninvasive technique for evaluation of left ventricular relaxation and estimation of filling pressures. J Am Coll Cardiol 1997;30:1527-33.

11 Sundereswaran L, Nagueh SF, Vardan S, et al. Estimation of left and right ventricular filling pressures after heart transplantation by tissue Doppler imaging. Am J Cardiol 1998;82:352-7.

12 Nageh MF, Kopelen HA, Zoghbi WA, et al. Estimation of mean right atrial pressure using tissue Doppler imaging. Am J Cardiol 1999;84:1448-51.

13 Mori K, Hayabuchi Y, Kuroda Y, et al. Left ventricular wall motion velocities in healthy children measured by pulsed wave Doppler tissue echocardiography: normal values and relation to age and heart rate. J Am Soc Echocardiogr 2000;13:1002-13

14 Bland JM, Altman DG. Statistical methods for assessing agreement between two methods of clinical measurement. Lancet 1986;i:307-10.

15 Kukulski T, Hubbert L, Arnold $M$, et al. Normal regional right ventricular function and its change with age: a Doppler myocardial imaging study. J Am Soc Echocardiogr 2000; 13:194-204.

16 Nagueh SF, Sun H, Kopelen HA, et al. Hemodynamic determinants of the mitral annulus diastolic velocities by tissue Doppler. J Am Coll Cardiol $2001 ; 37: 278-85$

17 Ichihashi K, Ewart P, Welmitz G, et al. Change in cardiac diastolic function in neonates. Heart Vessels 1997;12:216-20.

18 Frommelt PC, Ballweg JA, Whitstone BN, et al. Usefulness of Doppler tissue imaging analysis of tricuspid annular motion for determination of right ventricular function in normal infants and children. Am J Cardiol 2002;89:610-3.

19 Harada K, Tamura M, Yasuoka K, et al. A comparison of tissue Doppler imaging and velocities of transmitral flow in children with elevated left ventricular preload. Cardiol Young 2001;11:261-8.

\section{FROM BMJ JOURNALS}

\section{Factors involved in deciding to start preventive treatment: qualitative study of clinicians' and lay people's attitudes}

Please visit the Heartwebsite [wmw.heartinl. com] for a link to the full text of this article.

\section{David K Lewis, Jude Robinson, Ewan Wilkinson}

Objectives: To explore the views of clinicians and lay people about the minimum benefit needed to justify drug treatment to prevent heart attacks, and to explore the rationale behind treatment decisions.

Design: Qualitative study using semi-structured interviews.

Participants: 4 general practitioners, 4 practice nurses, and 18 lay people.

Setting: 8 general practices and 6 community settings across Liverpool.

Results: Participants varied widely in the minimum acceptable benefits chosen. Most people found the concepts difficult initially, and few appreciated that increased length of treatment should increase absolute benefits. Lay people usually wanted to make decisions for themselves, and clinicians supported this. Participants wanted to consider adverse effects and costs of treatment. Dislike of drug taking was common, and many people preferred lifestyle change to an imperfect treatment. Quality of life and personal views were more important than an individual's age.

Conclusions: Evidence based guidelines make assumptions about people's preferences, and, by using 10 year estimates of risk, inflate the apparent benefits of treatment. It is unlikely that guidelines could incorporate the wide range of people's preferences, and true dialogue is necessary between clinicians and patients before starting long term preventive treatment. 